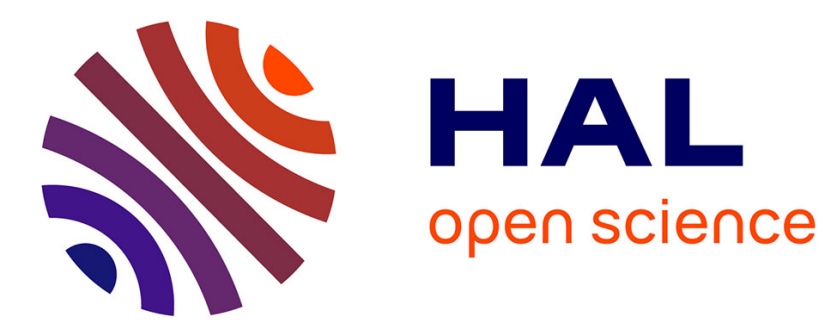

\title{
L'électrisation du cyclohexane en écoulement laminaire dans des tubes métalliques. I. Cas des solutions de picrate de triisoamylammonium
}

\author{
J.P. Gosse, A. Solofomboahangy, B. Gosse, A. Denat
}

\section{To cite this version:}

J.P. Gosse, A. Solofomboahangy, B. Gosse, A. Denat. L'électrisation du cyclohexane en écoulement laminaire dans des tubes métalliques. I. Cas des solutions de picrate de triisoamylammonium. Revue de Physique Appliquée, 1985, 20 (7), pp.493-502. 10.1051/rphysap:01985002007049300 . jpa00245362

HAL Id: jpa-00245362

https://hal.science/jpa-00245362

Submitted on 1 Jan 1985

HAL is a multi-disciplinary open access archive for the deposit and dissemination of scientific research documents, whether they are published or not. The documents may come from teaching and research institutions in France or abroad, or from public or private research centers.
L'archive ouverte pluridisciplinaire HAL, est destinée au dépôt et à la diffusion de documents scientifiques de niveau recherche, publiés ou non, émanant des établissements d'enseignement et de recherche français ou étrangers, des laboratoires publics ou privés. 


\title{
L'électrisation du cyclohexane en écoulement laminaire dans des tubes métalliques. I. Cas des solutions de picrate de triisoamylammonium
}

\author{
J. P. Gosse, A. Solofomboahangy, B. Gosse et A. Denat \\ Laboratoire d'Electrostatique et de Matériaux Diélectriques, C.N.R.S., avenue des Martyrs, 166X, \\ 38042 Grenoble Cedex, France
}

(Reçu le 27 novembre 1984, révisé le 26 mars 1985, accepté le 2 avril 1985)

\begin{abstract}
Résumé. - L'électrisation du cyclohexane s'écoulant en régime laminaire dans un tube capillaire métallique est étudiée en fonction des paramètres : résistivité électrique du liquide, longueur et diamètre du tube, nombre de Reynolds de l'écoulement. On discute la notion de potentiel zéta en tenant compte de l'influence de la force-image sur la distribution de charge dans la double couche à l'équilibre thermodynamique. On montre comment des mesures d'électrisation peuvent fournir le potentiel zéta $\zeta$ pour des liquides très peu conducteurs. Dans les solutions de TiAP dans le cyclohexane $\left(\sigma \sim 10^{-11} \Omega^{-1} \mathrm{~m}^{-1}\right), \zeta$ est trouvé égal à $+18 \mathrm{mV}$.
\end{abstract}

\begin{abstract}
The electrification of $\mathrm{CH}$ in laminar flow in a metallic tube has been studied as a function of different parameters : electrical resistivity, length and diameter of the tube, Reynolds number. The signification of the $\zeta$ potential is discussed, taking into account the influence of the image-force on the distribution of charge in the double layer at thermodynamic equilibrium. The determination of $\zeta$ is possible in resistive liquids $\left(>10^{11} \Omega \mathrm{m}\right)$ by studying the transient of electrification. For solutions of TiAP en $\mathrm{CH}, \zeta$ is $+18 \mathrm{mV}$.
\end{abstract}

\section{Introduction.}

L'électrisation d'hydrocarbures s'écoulant dans des tubes métalliques est un phénomène observé et étudié depuis longtemps : les premières études remontent à 1893 [1]. D'excellents articles de revue par Klinkenberg et Van der Minne [2], Schön [3], Gibson [4], Strawson and Lyle [5], Léonard [6], fournissent de précieux renseignements sur les connaissances acquises dans ce domaine, en particulier par l'Industrie Pétrolière.

Il est d'une grande importance économique de maîtriser ce phénomène qui a provoqué et provoque encore des explosions et des incendies dans des réservoirs recevant des liquides après leur passage dans des canalisations ou à travers des filtres. Des solutions pratiques ont été préconisées pour diminuer les risques d'incidents causés par l'électrisation; nous citerons le travail de Van der Minne et Klinkenberg [2] qui vers les années 1955 ont recherché des additifs permettant d'abaisser la résistivité du liquide en dessous d'un seuil critique (environ $10^{11} \Omega \mathrm{m}$ ), quand on les dissout en faible quantité $\left(<10^{-3}\right.$ mole $\left.1^{-1}\right)$ dans les hydrocarbures.

Pourtant, le phénomène n'est pas encore complè- tement compris : l'électrisation d'un liquide par écoulement est unanimement attribuée à l'entraînement d'une partie de la double couche électrique existant au contact métal-liquide; mais, on connaît mal les caractéristiques de cette couche ainsi que le mécanisme de régénération des charges emportées par le liquide. En 1977, à la Conférence sur l'électricité statique à Grenoble, Bright [7] citait des expériences non expliquées d'électrisation de kérosène entraînant des charges en densité volumique très différente suivant les additifs utilisés. Il indiquait que la mesure de la charge convectée est préférable au calcul quand des conditions de sécurité demandent l'évaluation de cette grandeur.

L'objet de notre travail a été d'améliorer la connaissance de l'interface chargée métal-liquide hydrocarbure et celle de l'électrisation des liquides lors de leur écoulement dans des tubes métalliques. Cette étude venait en complément d'une étude antérieure [8] qui a montré le rôle important joué par la double couche électrique dans la conduction électrique des hydrocarbures; en particulier, on observe souvent que leur conduction électrique est assurée par des ions créés à l'interface métal-liquide. 
A la différence de la plupart des travaux antérieurs, nous avons choisi un écoulement laminaire du liquide dans un tube de section circulaire; les résultats expérimentaux sont alors beaucoup plus faciles à exploiter qu'en régime turbulent. En effet, dans une section du tube située à une distance suffisante de l'entrée, les distributions de charge avec et sans écoulement laminaire sont identiques puisqu'il n'y a pas interaction entre la distribution des vitesses et celle de la charge d'espace. On peut ainsi atteindre la distribution de charge dans le liquide sans faire d'hypothèses sur la distribution de vitesse.

Nous prenons en compte la force-image et analysons son influence sur la double couche en milieu hydrocarbure [9]; à partir de ces résultats, nous discutons la signification des mesures d'électrisation et en particulier du potentiel zéta auquel il est fait référence dans les phénomènes électrocinétiques [10].

Nous avons voulu étudier de manière systématique l'influence de la résistivité du liquide sur l'électrisation et sur la double couche; en conséquence, les études expérimentales portent sur un hydrocarbure, le cyclohexane, contenant des quantités connues d'électrolyte; les solutions sont donc bien définies et leur résistivité varie entre $7 \times 10^{7} \Omega \mathrm{m}$ et $2,6 \times 10^{12} \Omega \mathrm{m}$.

Nous développerons dans le Chapitre 1 la théorie de l'électrisation des liquides en écoulement laminaire dans un tube de section circulaire, en précisant le rôle de la force-image sur la distribution de charge près du métal et celui de l'étendue de la double couche dans le liquide. Nous constaterons dans le Chapitre 2 que les études de ce phénomène en régime laminaire sont très peu nombreuses et que l'influence des additifs a été étudiée en régime turbulent. Après avoir décrit dans le Chapitre 3 les techniques expérimentales et les caractéristiques des produits utilisés, nous décrivons dans le Chapitre 4 les résultats de notre étude expérimentale de l'électrisation du cyclohexane contenant du picrate de triisoamylammonium.

1. La double couche électrique et l'électrisation d'un liquide par écoulement dans un tube de section circulaire.

1.1 LA DOUBLE COUCHE ÉLECTRIQUE À L'INTERFACE MÉTAL-LIQUIDE À L'ÉQUILIBRE THERMODYNAMIQUE. La double couche électrique telle qu'elle a été décrite par Stern, est caractérisée par deux longueurs : l'épaisseur $x_{A}$ de la couche compacte et la longueur de Debye $L_{\mathrm{D}}$ donnant l'étendue de la partie diffuse de la double couche [11].

La longueur $x_{\mathrm{A}}$ correspond à la distance minimum d'approche du métal par les ions, elle est de l'ordre de grandeur du rayon ionique; pour des ions ammonium quaternaire, $x_{\mathrm{A}}$ a été évaluée à 3 ou $4 \AA$.

La couche diffuse s'étend beaucoup plus loin dans le liquide $(\sim \mu \mathrm{m}$ pour les liquides non polaires) et d'autant plus loin que celui-ci est moins conducteur. Son étendue est caractérisée par $L_{\mathrm{D}}=\left(\varepsilon \phi_{0} / 2 q_{0}\right)^{1 / 2}$ où $\phi_{0}=k T / e_{0}=0,025 \mathrm{~V}$ à $T=300 \mathrm{~K}$ et $q_{0}$ est la densité volumique de charges négatives ou positives dans le liquide loin du métal.

Dans les liquides de faible permittivité, il faut ajouter à ces deux longueurs, la distance $x_{\mathrm{B}}$ qui caractérise l'étendue de la zone d'influence de la force-image (Fig. 1). En effet, à la distance $x$ d'une paroi métallique le potentiel de force-image est $e_{0} / 16 \pi \varepsilon x\left(e_{0}\right.$ est la charge de l'électron). Ce potentiel sera ressenti par un ion s'il est supérieur à $\phi_{0}=k T / e_{0}$, donc dans une couche contigue au métal et d'épaisseur $x_{\mathrm{B}}=e_{0}^{2} /$ $16 \pi \varepsilon k T$. Pour un liquide de permittivité relative $\varepsilon_{\mathrm{r}}=2$, à la température ambiante, $x_{\mathrm{B}}$ vaut $72 \AA$. Pour un liquide polaire comme l'eau $\left(\varepsilon_{\mathrm{r}}=80\right), x_{\mathrm{B}}$ est inférieure à $2 \AA$, donc inférieure à la distance minimum d'approche $x_{\mathrm{A}}$. Il est normal que la distance $x_{\mathrm{B}} \mathrm{n}$ 'apparaisse pas dans le modèle de Stern basé sur des expériences en milieu aqueux.

Par contre, dans les liquides de faible permittivité, il y a lieu de distinguer trois zones dans la double couche car la distribution de charge pour $x<x_{\mathrm{B}}$ sera fortement perturbée par rapport à la théorie classique.

L'influence de la force-image à l'interface métalliquide faiblement polaire est analogue à celle de la force de Coulomb dans ces mêmes liquides [12]. La force de Coulomb cause l'association ion-ion, la forceimage cause l'association ion-métal. Et les trois longueurs qui caractérisent la couche chargée près d'un métal ont leurs homologues dans l'atmosphère ionique entourant un ion dans le liquide.

A $x_{\mathrm{A}}$ correspond la distance $a$ centre à centre des ions associés et formant une paire, $x_{\mathrm{A}}=a / 2$ environ. A $x_{\mathrm{B}}$ correspond la longueur de Bjerrum $l_{\mathrm{B}}$ définie par $e_{0} / 8 \pi \varepsilon l_{\mathrm{B}}=k T / e_{0}, x_{\mathrm{B}}=l_{\mathrm{B}} / 2$. Selon la théorie de Bjerrum, si $l_{\mathrm{B}} \gg a$ il y a association ionique. Dans le cas contraire, les ions restent libres.
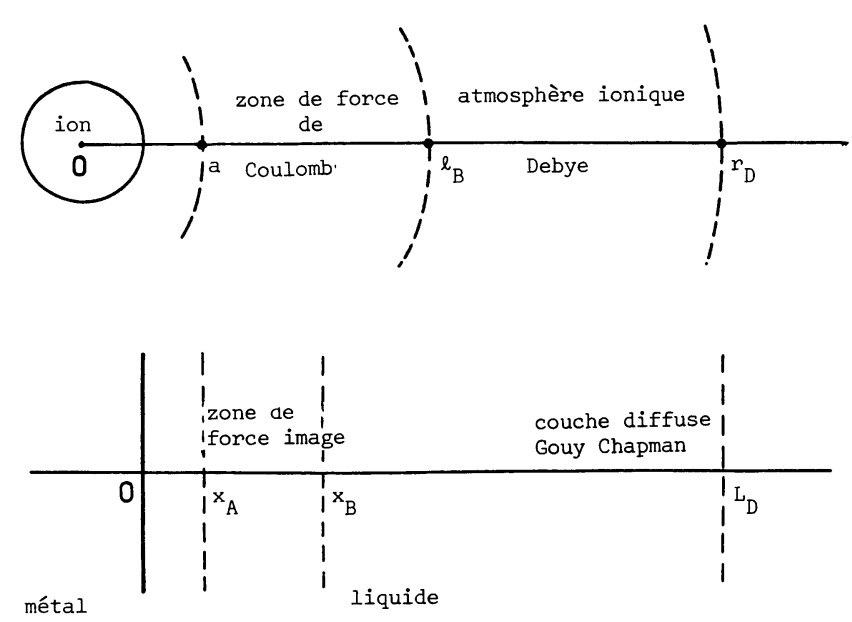

Fig. 1. - Double couche à l'interface métal/liquide et atmosphère ionique autour d'un ion (cas d'un liquide faiblement polaire).

[The double layer at the metal/non polar liquid interface (A) and the ionic atmosphere around a central ion (B).] 
A $L_{\mathrm{D}}$ correspond le rayon de l'atmosphère ionique $r_{\mathrm{D}}, L_{\mathrm{D}}=r_{\mathrm{D}}$.

1.2 DENSITÉ DE CHARGE À L'INTERFACE MÉTAL-LIQUIDE À L'ÉQUILIBRE THERMODYNAMIQUE. - Soit $q(x)$ la densité volumique de charge à la distance $x$ du métal.

Considérons d'abord la région de force-image $x<x_{\mathrm{B}}$. On peut écrire :

$$
\left[E-e_{0} / 16 \pi \varepsilon x^{2}\right] q-\phi_{0} \mathrm{~d} q / \mathrm{d} x=0
$$

$E$ est le champ électrique.

On peut montrer que dans cette région $E(x) \ll$ $e_{0} / 16 \pi \varepsilon x^{2}$ [13]. L'équation (1) simplifiée s'écrit :

$$
x_{\mathrm{B}} q / x^{2}+\mathrm{d} q / \mathrm{d} x=0 \text {. }
$$

Son intégration donne

$$
q(x)=q_{\mathrm{B}} \exp \left[\left(x_{\mathrm{B}} / x\right)-1\right] .
$$

L'équation (2) nous permet de calculer la densité volumique de charge $q_{\mathrm{n}}$ à la distance minimum d'approche $x_{\mathrm{A}}$ de la paroi, $q_{\mathrm{p}}=q_{\mathrm{B}} \exp \left[\left(x_{\mathrm{B}} / x_{\mathrm{A}}\right)-1\right]$. Pour $\varepsilon_{\mathrm{r}}=2, x_{\mathrm{B}}=72 \AA, x_{\mathrm{A}}=5 \AA$ on obtient $q_{\mathrm{p}}=$ $6,6 \times 10^{5} q_{\mathrm{B}}$.

Cette évaluation numérique montre combien la force-image renforce la densité volumique de charges près du métal.

En dehors de la zone de force-image $\left(x>x_{\mathrm{B}}\right)$, les distributions de charges positives et négatives et du potentiel $\phi$ dans la double couche s'obtiennent à partir de l'équation de Poisson et de l'équation de Boltzmann.

On obtient pour la charge d'espace $q=q_{+}-q_{-}$

$$
q=q_{0}\left[\exp \left(-\phi / \phi_{0}\right)-\exp \left(\phi / \phi_{0}\right)\right] .
$$

On peut ainsi calculer la densité volumique de charge nette $q_{\mathrm{B}}$ juste en dehors de la zone de force-image, en fonction de $\phi_{\mathrm{B}}=\phi\left(x=x_{\mathrm{B}}\right)$.

La densité volumique de charge $q_{\mathrm{p}}$ au voisinage du métal $\left(x=x_{\mathrm{A}}\right)$ s'obtient en fonction de $q_{0}$ en raccordant les deux distributions, soit

$$
\begin{aligned}
q_{\mathrm{p}}=\left[\exp \left[\left(x_{\mathrm{B}} / x_{\mathrm{A}}\right)-1\right]\right] q_{0}\left[\exp \left(-\phi_{\mathrm{B}} / \phi_{0}\right)\right. \\
\left.-\exp \left(\phi_{\mathrm{B}} / \phi_{0}\right)\right] .
\end{aligned}
$$

\subsection{Densité VolumiQue DE CHARGE CONVECTÉe PAR} LE LIQUIDE EN ÉCOULEMENT LAMINAIRE. CAS $L_{\mathrm{D}} \ll R$. - Le calcul de la densité volumique $q_{\mathrm{v}}$ de charge convectée est classique. On considère un tube métallique de très grande longueur dont le rayon $R$ satisfait $R \gg L_{\mathrm{D}}$, l'écoulement du liquide y est laminaire.

La vitesse n'ayant pas de composante radiale, la distribution de la charge d'espace dans le tube est la même pour un liquide en repos ou en mouvement.

Le courant convecté s'écrit $I=\int_{0}^{R} q(r) u(r) 2 \pi r \mathrm{~d} r$, $u(r)$ est la vitesse à la distance $r$ de l'axe.
La zone chargée influencée par la force-image $\left(x<x_{\mathrm{B}}\right)$ contribue de façon négligeable à l'électrisation [13]. En effet, l'augmentation très rapide de la densité volumique de charges se produit dans une couche de très faible épaisseur dans laquelle la vitesse du fluide est aussi très faible. L'intégrale peut donc être arrêtée à $r=R-x_{\mathrm{B}}$. La densité $q_{\mathrm{v}}$ de charge convectée (courant $I /$ débit de liquide) s'en déduit :

$$
q_{\mathrm{v}}=8 \varepsilon \phi_{\mathrm{B}} / R^{2}
$$

avec $\phi_{\mathrm{B}}=\phi_{0} \log \left\{q_{\mathrm{B}} / 2 q_{0}+\left[1+\left(q_{\mathrm{B}} / 2 q_{0}\right)^{2}\right]^{1 / 2}\right\}$.

L'expression de $q_{\mathrm{v}}$ se simplifie dans deux cas limites

$$
\begin{array}{ll}
\phi_{\mathrm{B}} / \phi_{0} \gg 1 \quad \text { alors } & q_{\mathrm{v}}=8 \varepsilon \phi_{0}\left(\log q_{\mathrm{B}} / q_{0}\right) / R^{2} \\
\phi_{\mathrm{B}} / \phi_{0} \ll 1 & q_{\mathrm{v}}=4 \varepsilon \phi_{0} q_{\mathrm{B}} / q_{0} R^{2} .
\end{array}
$$

et

La figure 2 donne les variations de $q_{\mathrm{v}}$ en fonction de $q_{\mathrm{B}}$ pour différentes résistivités du cyclohexane (mobilité ionique : $1,4 \times 10^{-8} \mathrm{~m}^{2} \mathrm{~V}^{-1} \mathrm{~s}^{-1}$ ) s'écoulant dans un tube de $0,5 \mathrm{~mm}$ de rayon. Les valeurs correspondant à $\phi_{\mathrm{B}} \sim \phi_{0}$ ont été obtenues par résolution numérique du problème [14].

L'interprétation antérieure de l'électrisation basée sur la représentation de la double couche due à Stern donne

$$
q_{\mathrm{v}}=8 \varepsilon \zeta / R^{2} .
$$

Les mesures d'électrisation par écoulement d'un liquide fournissent la valeur d'un potentiel appelé potentiel zéta. Nous montrons (voir (5) et (6)) que le potentiel $\zeta$ déterminé par ces expériences est le potentiel à la distance $x_{\mathrm{B}}=e_{0}^{2} / 16 \pi \varepsilon k T$ du métal. La densité volumique de charge en $x=x_{\mathrm{B}}$ est $q_{\mathrm{B}}$, la densité de charge $q_{\mathrm{p}}$ en $x=x_{\mathrm{A}}$ qui s'en déduit par la relation (2), est beaucoup plus élevée que $q_{\mathrm{B}}$. Dans la suite nous conserverons pour $\phi_{B}$ la notation habituelle $\zeta$.

1.4 Densité Volumique DE ChaRge CONVECTÉe EN ÉCOUlement Laminaire. CAS $L_{\mathrm{D}} \sim R$. - Dans le cas assez fréquent où la condition $R \gg L_{\mathrm{D}}$ n'est pas réalisée (résistivité élevée), seul le calcul numérique permet d'atteindre $q_{\mathrm{v}}$; le traitement mathématique a été décrit par Touchard [15].

Les résultats de ces calculs sont présentés sur la figure 3.

La densité $q_{\mathrm{v}}$ de charge convectée est donnée en fonction du rayon du tube pour une résistivité donnée (donc $L_{\mathrm{D}}$ et $q_{0}$ données) et une densité volumique de charges $q_{\mathrm{B}}$ constante et égale à $10^{-3} \mathrm{Cm}^{-3}$. Le rapport $q_{\mathrm{B}} / q_{0}$ et le potentiel $\zeta$ restent donc constants quand le rayon $R$ du tube varie.

On constate que tant que $R / L_{\mathrm{D}}>10, q_{\mathrm{v}}$ varie comme $R^{-2}$; pour $R / L_{\mathrm{D}}<1, q_{\mathrm{v}}$ est voisine de la densité volumique de charges à la paroi considérée.

Ces résultats peuvent être aussi présentés sur la figure 4 où nous donnons les variations de $Q=$ $q_{\mathrm{v}} /\left(8 \varepsilon \zeta / R^{2}\right)$ en fonction du rapport $L_{\mathrm{D}} / R$ (ou de la 


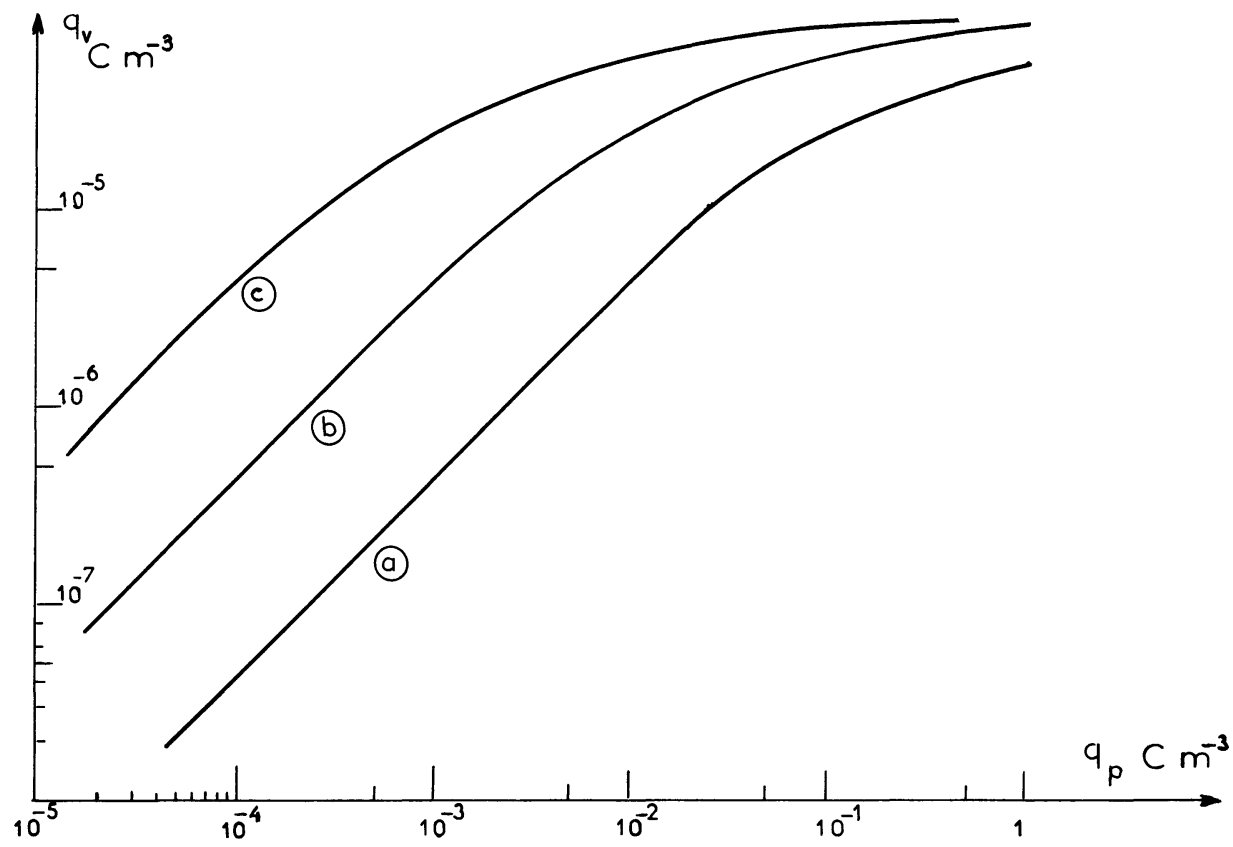

Fig. 2. - Densité volumique moyenne de charge convectée $q_{\mathrm{v}}$ en fonction de la densité volumique de charge $q_{\mathrm{p}}$, à la distance $x_{\mathrm{A}}$ du métal. Cas du cyclohexane $\varepsilon_{\mathrm{r}}=2$; mobilité ionique $=1,4 \times 10^{-8} \mathrm{~m}^{2} \mathrm{~V}^{-1} \mathrm{~s}^{-1}$; résistivité : courbe a $=0,3 \times$ $10^{10} \Omega \mathrm{m}$, courbe $\mathrm{b}=0,3 \times 10^{11} \Omega \mathrm{m}$, courbe $\mathrm{c}=0,3 \times 10^{12} \Omega \mathrm{m}$. Rayon intérieur du tube $=0,5 \mathrm{~mm}$. Les calculs correspondant à $\phi / \phi_{0} \sim 1$ ont été effectués par G. Touchard.

[Average convected charge density $q_{\mathrm{v}}$ as a function of the charge density $q_{\mathrm{p}}$ at the distance $x_{\mathrm{A}}$ from the metal. Cyclohexane $\left(\varepsilon_{\mathrm{r}}=2\right)$, ionic mobility $=1.4 \times 10^{-8} \mathrm{~m}^{2} \mathrm{~V}^{-1} \mathrm{~s}^{-1}$. Resistivity : $0.3 \times 10^{10} \Omega \mathrm{m}(\mathrm{a}), 0.3 \times 10^{11} \Omega \mathrm{m}(\mathrm{b}), 0.3 \times 10^{12} \Omega \mathrm{m}(\mathrm{c})$. Tube radius : $0.5 \mathrm{~mm}$. The numerical calculations for $\phi / \phi_{0} \sim 1$ have been made by G. Touchard.]

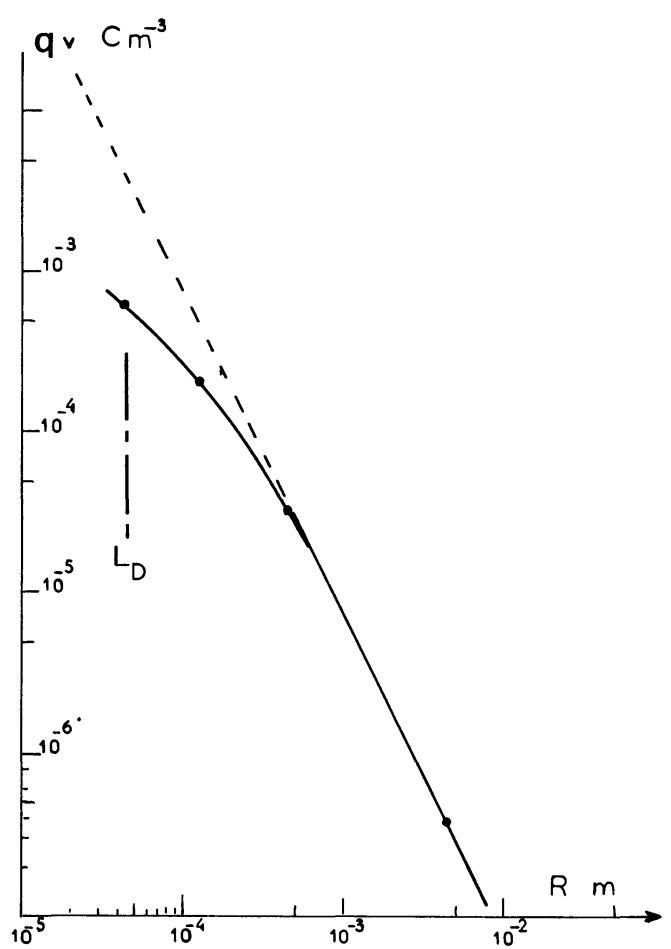

Fig. 3. - Densité volumique de charge convectée $q_{\mathrm{v}}$ en fonction du rayon intérieur du tube. Cyclohexane de résistivité $3 \times 10^{11} \Omega \mathrm{m} ; q_{\mathrm{B}}=10^{-3} \mathrm{C} \mathrm{m}^{-3}$.

[Tube radius dependence of the convected charge density $q_{\mathrm{v}}$. Cyclohexane resistivity : $3 \times 10^{11} \Omega \mathrm{m}, q_{\mathrm{B}}=10^{-3} \mathrm{C} \mathrm{m}^{-3}$.]

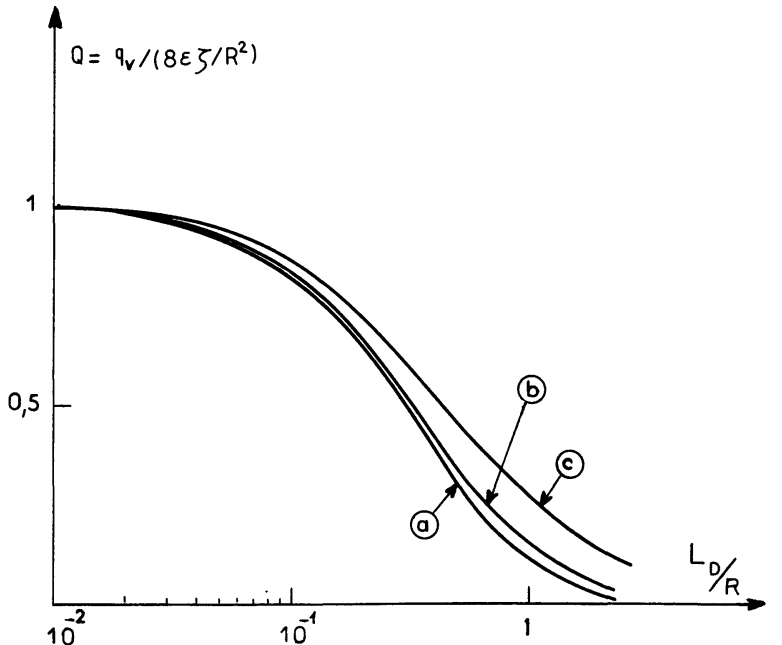

Fig. 4. - Densité réduite de charge convectée $q_{v} /\left(8 \varepsilon \zeta / R^{2}\right)$ en fonction du rapport $L_{\mathrm{D}} / R$. Calculs effectués par $\mathrm{G}$. Touchard pour différents rapports $q_{\mathrm{B}} / q_{0}$; courbe a $=0,9$; courbe $b=9$; courbe $c=90$.

[Reduced convected charge density $Q$ as a function of the ratio $L_{\mathrm{D}} / R$. Ratio $q_{\mathrm{B}} / q_{0}=0.9(\mathrm{a}), 9(\mathrm{~b}), 90$ (c). $]$

résistivité du cyclohexane pour $R=0,5 \mathrm{~mm}$ ) pour différentes valeurs du rapport $q_{\mathrm{B}} / q_{0}$. Le potentiel $\zeta$ intervenant dans le calcul de $Q$ est défini et constant pour une valeur donnée de $q_{\mathrm{B}} / q_{0}$. On constate que 
pour $L_{\mathrm{D}} \ll R$, on obtient $Q \# 1$, soit $q_{\mathrm{v}} \# 8 \varepsilon \zeta / R^{2}$; pour $L_{\mathrm{D}}=R$ et la valeur de $q_{\mathrm{B}} / q_{0}=9$ (courbe 2 ), on obtient $q_{\mathrm{v}}=0,16\left(8 \varepsilon \zeta / R^{2}\right)$. Pour $L_{\mathrm{D}} \gg R$, la densité volumique $q_{\mathrm{v}}$ de charge convectée tend vers la densité volumique $q_{\mathrm{B}}$ constante de charges à la paroi, et $Q$ tend vers zéro.

Cette courbe permet de déterminer le rapport $q_{\mathrm{B}} / q_{0}$ et donc $q_{\mathrm{B}}$ à partir des mesures de la densité $q_{\mathrm{v}}$ de charge convectée et de la résistivité du liquide; on connaît donc le rapport $L_{\mathrm{D}} / R ; q_{\mathrm{B}} / q_{0}$ qui satisfait l'équation $q_{\mathrm{v}}=Q\left(\frac{L_{\mathrm{D}}}{R}, \frac{q_{\mathrm{B}}}{q_{0}}\right) \cdot 8 \varepsilon \zeta\left(q_{\mathrm{B}} / q_{0}\right) / R^{2}$, peut être calculée par itérations successives à l'aide de la figure 4.

\section{Travaux antérieurs.}

Les études d'électrisation de liquide en écoulement laminaire sont peu nombreuses et, à notre connaissance, la formule de base du phénomène $q_{\mathrm{v}}=8 \varepsilon \zeta / R^{2}$ $a$ fait l'objet de très peu de vérifications. Nous citerons Rutgers, De Smet et De Myer [16] qui ont étudié des solutions $10^{-5}, 10^{-4}$ et $10^{-3}$ mole $1^{-1}$ de diisopropylsalicylate de zinc dans du benzène. Les tubes capillaires en verre de $1 \mathrm{~m}$ de longueur avaient pour rayon $0,044 \mathrm{~cm}, 0,116 \mathrm{~cm}$ et $0,188 \mathrm{~cm}$. La densité $q_{\mathrm{v}}$ de charges convectées variait en $R^{-2}$ comme il est prévu par la théorie, le potentiel $\zeta$ correspondant aux solutions $10^{-5}$

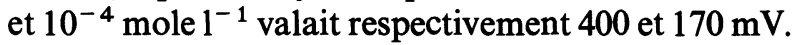

Gibbings [17] a étudié l'électrisation de kérosène en régime laminaire dans des tubes en acier inoxydable, de rayon intérieur $3,48 \mathrm{~mm}$ et de longueur variable mais inférieure à $1,5 \mathrm{~m}$. La densité de charges convectées variait avec le nombre de Reynolds contrairement à la théorie; la résistivité du liquide n'est pas fournie. Gibbings interprète ses expériences par une électrisation du liquide à son entrée du tube, masquant le phénomène se produisant dans le tube lui-même. Il suppose donc que la longueur du tube était insuffisante pour obtenir une double couche à l'équilibre dans la section de sortie du tube.

Enfin, plus récemment, Touchard $[18,19]$ a étudié l'électrisation de l'heptane s'écoulant dans des tubes d'acier inoxydable de $1 \mathrm{~m}$ de longueur, de rayon intérieur variable et inférieur à $2 \mathrm{~mm}$. Le potentiel $\zeta$ correspondant se trouve dans la gamme 80 à $120 \mathrm{mV}$; toutefois, dans ces expériences, la densité de charges convectées ne varie pas en $R^{-2}$ pour des rayons du tube supérieurs à $0,04 \mathrm{~cm}$.

L'influence d'additifs au liquide sur son électrisation a été par contre beaucoup plus étudiée, mais en écoulement turbulent du liquide, ce qui correspond aux conditions réelles d'électrisation des hydrocarbures. Les études ont été pour la plupart simplement descriptives, l'interprétation ne pouvant se faire qu'après résolution numérique du problème, ce qui n'a été fait que par Touchard [18] à notre connaissance.

Depuis les études de Goodfellow et Graydon [20], de Luus [21], il est généralement admis que des additifs neutres, non dissociés en principe, ne modifient l'électrisation du liquide que dans la mesure où leur dissolution s'accompagne d'une augmentation de la conductivité.

L'étude de base de l'influence d'additifs dissociés reste celle de Klinkenberg et Van der Minne [2]. Deux types de composés ont été étudiés : d'une part, les sels d'un mélange d'acide mono- et dialkylsalicylique (en particulier diisopropyl), le cation bi- ou trivalent étant métallique (aluminium, chrome, calcium); d'autre part le di-2-éthylhexylsulphosuccinate de sodium (Aérosol OT), des inhibiteurs de corrosion, des asphaltènes qui ont des propriétés surfactantes. Klinkenberg et Van der Minne [2] ont également fait une étude de l'électrisation en fonction de la résistivité du liquide. Sur la figure 5 donnée à titre d'exemple apparaissent les variations de la densité volumique de charge convectée par de l'essence contenant du sel de chrome de l'acide salycilique $(\mathrm{Cr} \mathrm{AC})$ en fonction de sa conductivité La densité de charge convectée passe par une valeur maximum de $3 \times 10^{-5} \mathrm{C} \mathrm{m}^{-3}$ pour une conductivité de $5 \times 10^{-11} \Omega^{-1} \mathrm{~cm}^{-1}$. La décroissance de $q_{\mathrm{v}}$ observée aux résistivités élevées est attribuée à la longueur insuffisante du tube, la double couche n'étant pas complètement formée à l'extrémité du tube. La courbe 2 (Fig. 5) est obtenue à partir de la courbe 1 en extrapolant les résultats à une longueur infinie du tube, d'après une hypothèse de Schön. La décroissance de la charge convectée aux conductivités faibles n'est pas expliquée. Ces études ont permis à Klinkenberg et Van der Minne de montrer qu'il suffit d'une faible addition d'un électrolyte tel que le $\mathrm{Cr}$ AC ( $\lesssim 10^{-6}$ mole $^{-1}$ ) conférant à l'essence une conductivité inférieure à $10^{-11} \Omega^{-1} \mathrm{~m}^{-1}$, pour obtenir une densité de charge convectée inférieure à $10^{-6} \mathrm{C} \mathrm{m}^{-3}$,

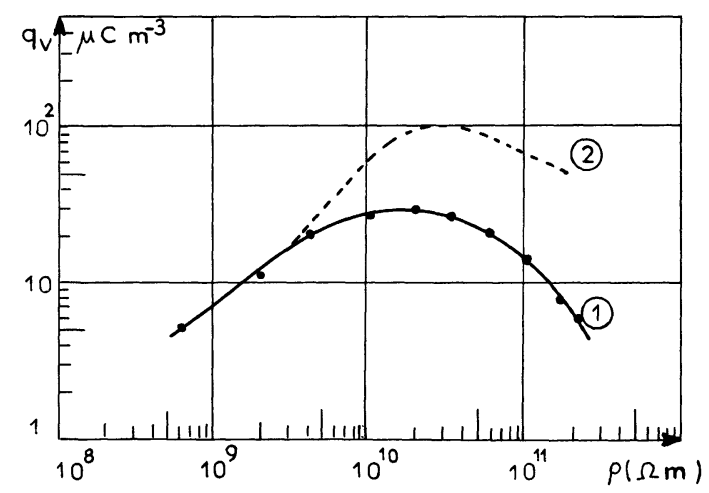

Fig. 5. - Densité volumique de charge convectée par des mélanges de pétrole brut du Koweit et d'essence en fonction de la résistivité du liquide, d'après Klinkenberg et Van der Minne [2]. $R_{\mathrm{e}}=3000$; diamètre intérieur du tube $=3 \mathrm{~mm}$. Courbe 1 : résultats expérimentaux : longueur du tube $=$ $0,5 \mathrm{~m}$. Courbe 2 : après correction, pour faire correspondre les résultats à une longueur infinie du tube.

[Resistivity dependence of the charge density convected by solutions of Kowait crude oil in gasoline (after Ref. [2]). $R_{\mathrm{e}}=3000$; tube radius $=3 \mathrm{~mm}$; tube length $=0.5 \mathrm{~m}$. Experimental results (1), values corrected for length (2). ] 
valeur de seuil de " sécurité complète » lors de transvasement ou d'écoulement d'hydrocarbures.

Dans cette bibliographie sommaire des principaux travaux sur l'influence d'additifs, nous devons citer les très nombreux essais de Léonard [22] sur l'électrisation de kérosène et du n-heptane s'écoulant à travers des filtres, notamment en papier. L'interprétation de ce type d'essais paraît à l'heure actuelle très difficile, compte tenu de la méconnaissance du milieu poreux qu'est le filtre et de celle de l'interface filtre-hydrocarbure.

En conclusion, les expériences d'électrisation de liquides dopés ont toutes été faites en écoulement turbulent sans, à quelques exceptions près, se préoccuper du paramètre "conductivité » fondamental dans ces études. Il en résulte qu'il n'y a pas d'interprétation théorique de l'influence des additifs.

\section{Techniques expérimentales.}

3.1 MéTHOde De MeSURES. - Le schéma du montage expérimental est donné sur la figure 6. Le liquide en (2) mis sous pression par le dispositif (1) s'écoule dans le tube capillaire après ouverture de la vanne (10). Les charges entraînées par le liquide sont recueillies dans un pot collecteur (5) soigneusement isolé. Le potentiel de ce collecteur est mesuré à l'aide d'un électromètre à haute impédance d'entrée $\left(\sim 10^{14} \Omega\right)$.

La densité volumique $q_{\mathrm{v}}$ de charges est calculée de la manière suivante : pendant un intervalle de temps $\mathrm{d} t$, un volume $\mathrm{d} v$ de liquide a entraîné une charge $\mathrm{d} q$, le potentiel du collecteur s'est élevé de $\mathrm{d} V$. Soit $C$ la capacité de l'ensemble collecteur, système de mesure, cage de Faraday, on a $\mathrm{d} V=\mathrm{d} q / C$ et $q_{\mathrm{v}}=\mathrm{d} q / \mathrm{d} v=$ $C\left(\frac{\mathrm{d} V}{\mathrm{~d} t}\right) / Q_{\mathrm{v}} \cdot q_{\mathrm{v}}$ s'obtient donc à partir de la capacité $C$, du débit de liquide $Q_{\mathrm{v}}$, de la pente $\mathrm{d} V / \mathrm{d} t$ de l'enregistrement de la tension du collecteur en fonction du temps.

3.2 DisPOSITIF EXPÉRIMENTAL. - Le dispositif expérimental est à peu près analogue à celui mis au point par Touchard [18]. Il comprend trois parties (Fig. 6) : le circuit hydraulique, le circuit de mise sous pression du liquide, les appareils de mesure.

Avant les expériences, le liquide remplit les boules de verre de volume calibré(4) et la réserve de liquide(2). A l'ouverture de la vanne (10), le liquide s'écoulant dans le tube capillaire (3) tombe dans le collecteur (5). Par l'intermédiaire de la vanne (6), on peut vider le collecteur et renvoyer le liquide dans la réserve (7). L'ensemble des pièces en contact avec le liquide sont en téflon, en verre pyrex, en acier inoxydable, les joints sont en viton.

Les tubes capillaires en acier inoxydable ont au plus $3 \mathrm{~m}$ de longueur et $1 \mathrm{~mm}$ de diamètre intérieur. L'extrémité d'entrée du tube capillaire est située sur l'axe d'un tube de rayon beaucoup plus grand $(9 \mathrm{~mm})$. Cette disposition permet d'éviter une électrisation éventuelle dans un convergent tout en ne perturbant pas l'écoulement sur une trop grande longueur $(50 D \sim 5$ à $10 \mathrm{~cm})$.

La capacité de l'ensemble collecteur-câble de connexion-électromètre déterminée par un pont de mesures est de $92 \mathrm{pF}$. Dans les expériences où le liquide était fortement chargé, une capacité supplémentaire a été montée en parallèle sur le pot collecteur pour que le potentiel du collecteur par rapport à la masse reste inférieur à quelques volts.

La conductivité du liquide dans le circuit hydraulique est mesurée dans une cellule contenant deux électrodes planes et parallèles, au moyen d'un pont

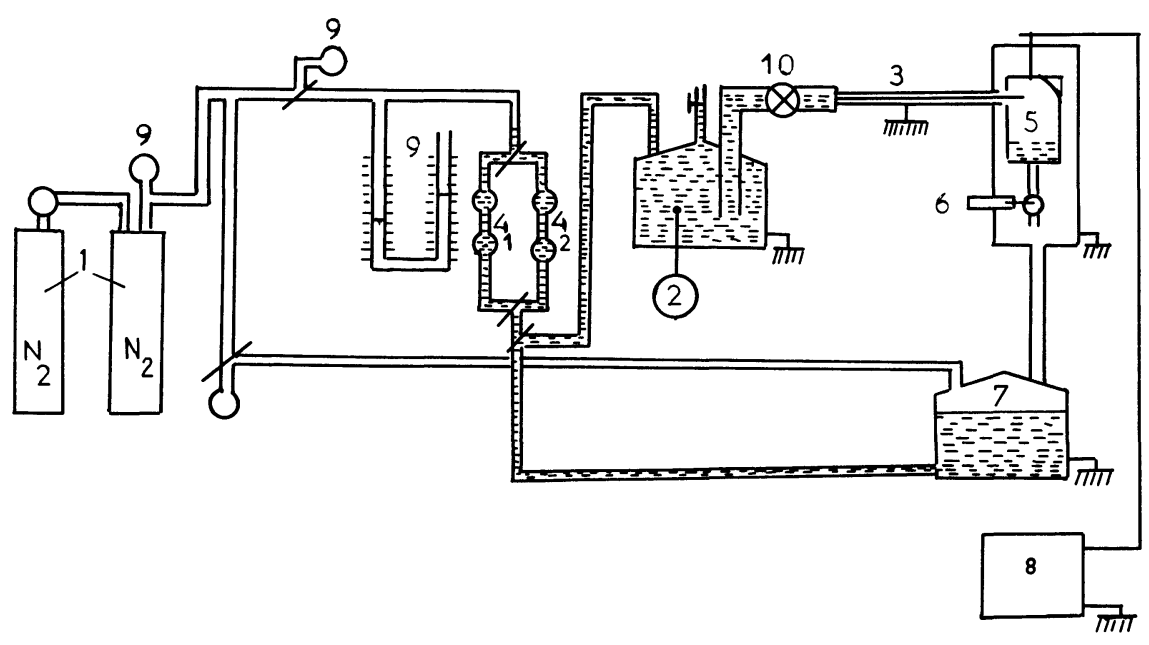

Fig. 6. - Dispositif expérimental. 1. Réservoirs d'azote sous pression. 2. Réserve de liquide. 3. Capillaire en acier inoxydable $l=1$ ou $3 \mathrm{~m}$. 4. Dispositif de mesure du débit de liquide. 5. Pot collecteur. 6. Vanne de vidange du collecteur. 7. Réserve de liquide. 8. Electromètre et enregistreur. 9. Mesure de pression. 10. Vanne de mise en écoulement du liquide.

[Experimental arrangement. 1. Compressed argon vessels. 2. Reservoir tank, 3. Stainless-steel capillary. 4. Flowmeter. 5. Receiving tank. 6. Valve. 7. Stainless-steel reservoir. 8. Electrometer and recorder. 9. Manometers. 10. Valve.] 
alternatif General Radio 1621. On opère à plusieurs fréquences et on vérifie que la conductance mesurée est indépendante de la fréquence.

3.3 CARACTÉRISTIQUES PHYSICO-CHIMIQUES DES PRODUITS ÉTUDIÉS. - Le cyclohexane, d'origine Merck, de qualité « spectroscopie ", a une permittivité relative de 2,023 , une viscosité de $1 \mathrm{cp}$ à $20^{\circ} \mathrm{C}$. Les solutés que nous avons utilisés, peuvent être classés en deux catégories :

1) Les solutés neutres : la tétraméthylphénylènediamine (TMPD) et la triisoamylamine (TIA). La dissolution de ces produits correctement purifiés n'entraîne pas d'augmentation de la conductivité du milieu, ce sont des composés facilement oxydables.

2) L'électrolyte : le picrate de triisoamylammonium (TiAP). Il est synthétisé et purifié d'après la méthode antérieurement décrite [23].

La solubilité du TiAP dans le cyclohexane est $7,4 \times 10^{-4}$ mole $1^{-1}$. C'est un électrolyte de type ionophore aux propriétés décrites par la théorie de Fuoss et Kraus [24]. Le TiAP est faiblement dissocié en milieu hydrocarbure; à saturation il confère au cyclohexane une résistivité de $2 \times 10^{11} \Omega \mathrm{m}$.

\section{Electrisation de solutions de TiAP dans le cyclo- hexane.}

Dans les expériences d'électrisation du cyclohexane contenant du TiAP (résistivité $3 \times 10^{11} \Omega \mathrm{m}$ ), pour un écoulement caractérisé par un nombre de Reynolds $R_{\mathrm{e}}=1000$, dans un tube de $3 \mathrm{~m}$ de long et de diamètre intérieur $2 R=1 \mathrm{~mm}$, les différentes grandeurs avaient les valeurs suivantes : vitesse sur l'axe du tube = $2,62 \mathrm{~m} \mathrm{~s}^{-1}$; temps de séjour dans le tube d'une particule liquide située sur l'axe du tube : $1,15 \mathrm{~s}$; longueur de Debye $L_{\mathrm{D}}$ caractérisant l'étendue de la couche diffuse $=43 \mu \mathrm{m} ;$ rapport $L_{\mathrm{D}} / R=8,6 \times 10^{-2}$; temps de relaxation $\varepsilon \rho$ du liquide $=5,3 \mathrm{~s}$; débit de liquide : $1,03 \times 10^{-6} \mathrm{~m}^{3} \mathrm{~s}^{-1}$. Dans ces conditions expérimentales, la densité volumique de charges convectées valait environ $10^{-5} \mathrm{C} \mathrm{m}^{-3}$; et l'intensité correspondante du courant était $10^{-11} \mathrm{~A}$. Ce courant est extrêmement petit, et si l'on assimile l'électrisation du liquide à un tri des ions du liquide par le métal, on peut dire que ce tri est d'une très faible efficacité. On entraîne par le liquide une charge nette inférieure à $10^{-5} \mathrm{C} \mathrm{m}^{-3}$, alors que la densité en ions positifs et négatifs contenue dans le liquide est d'environ $1,2 \times 10^{-4} \mathrm{C} \mathrm{m}^{-3}$, soit au moins 10 fois plus. La faiblesse du tri des ions par le métal rend l'étude délicate, les différents paramètres devant être bien contrôlés.

4.1 Signe De la Charge CONVECtée. - Le cyclohexane pur sans additif s'électrise positivement. Il en est de même pour les solutions cyclohexane + tétraméthylphénylènediamine (TMPD), cyclohexane + TiAP + benzène. Par contre, les solutions de TiAP dans le cyclohexane s'électrisent tantôt positivement, tantôt négativement. L'addition de triisoamylamine
(TIA) ou d'eau à ces solutions cause une charge convectée toujours négative.

Le signe de la charge convectée n'a pas reçu d'explication, il faudrait connaître les affinités pour le métal des différentes substances en solution et leur adsorption.

4.2 Densité volumique de Charge ConvectéE. De façon tout à fait générale, les résultats de mesures d'électrisation ne sont significatives et ne peuvent fournir des informations sur la structure de la double couche à l'interface métal-liquide que si la double couche dans la section de sortie du tube a la même distribution avec et sans écoulement de liquide. En effet, malgré quelques travaux tels que ceux de Koszman et Gavis [25], il n'existe pas de théorie satisfaisante permettant de calculer la densité de charge convectée dans un tube de longueur infinie à partir de la mesure de la densité de charge convectée dans un tube de longueur insuffisante. Une de nos préoccupations a été de définir les conditions expérimentales permettant d'atteindre la densité de charge convectée correspondant à une distribution stationnaire de charges dans la double couche, à la sortie du tube.

4.3 Influence de la longueur du tube. - Nos premiers essais avec des tubes de $1 \mathrm{~m}$ de long sur des solutions de résistivité élevée laissaient supposer que la double couche n'avait pas sa distribution stationnaire à l'extrémité du tube : $q_{\mathrm{v}}$ était une fonction décroissante de $R_{\mathrm{e}}$ en régime laminaire, sa valeur était très faible $\left(\sim \mu \mathrm{C} \mathrm{m}^{-3}\right)$. Nous avons poursuivi l'étude avec des tubes de 3 et $1 \mathrm{~m}$ de longueur issus d'une même fabrication. On constate (Tableau I) que pour une solution de résistivité $3,5 \times 10^{11} \Omega \mathrm{m}, q_{\mathrm{v}}$ augmente avec $R_{\mathrm{e}}$ et avec la longueur du tube. Par contre pour une solution de résistivité $4 \times 10^{10} \Omega \mathrm{m}, q_{\mathrm{v}}$ croît avec $R_{\mathrm{e}}$ mais ne dépend plus de la longueur du tube (Tableau II). Une étude plus approfondie nous a permis de montrer que même dans ce cas, on n'obtenait pas la valeur cherchée de $q_{\mathrm{v}}$.

4. 4 RéGime tRANSITOIRE D'ÉLECTRISATION. - Si l'on enregistre le potentiel du pot collecteur dès la mise sous pression du liquide, on constate que la densité de charge convectée $q_{\mathrm{v}}$ calculée à partir de la pente de la tangente à la courbe enregistrée $V(t)$ est une fonction

Tableau I. - Densité volumique de charge convectée par une solution de $\mathrm{CH}+\operatorname{TiAP}\left(\rho=3,5 \times 10^{11} \Omega \mathrm{m}\right)$.

[Average charge density convected by TIAP solutions in cyclohexane $\left(\rho=3.5 \times 10^{11} \Omega \mathrm{m}\right)$.]

\begin{tabular}{|c|c|c|}
\hline$R_{\mathrm{e}}$ & $1 \mathrm{~m}$ & $3 \mathrm{~m}$ \\
\hline 400 & $6,5 \times 10^{-7} \mathrm{C} \mathrm{m}^{-3}$ & $5,2 \times 10^{-6} \mathrm{C} \mathrm{m}^{-3}$ \\
\hline 1000 & $9 \times 10^{-8} \mathrm{C} \mathrm{m}^{-1}$ & $4,6 \times 10^{-6} \mathrm{C} \mathrm{m}^{-3}$ \\
\hline
\end{tabular}


Tableau II. - Densité volumique de charge convectée pour une solution $\mathrm{CH}+\mathrm{TiAP}+$ benzène $(\rho=4 \times$ $10^{10} \Omega \mathrm{m}$ ).

[Average charge density convected by TIAP solutions in a mixture cyclohexane-benzene $\left(\rho=4 \times 10^{10} \Omega \mathrm{m}\right)$.]

\begin{tabular}{|c|c|c|}
\hline$R_{\mathrm{e}}$ & $1 \mathrm{~m}$ & $3 \mathrm{~m}$ \\
\hline 400 & $0,6 \times 10^{-5} \mathrm{C} \mathrm{m}^{-3}$ & $0,65 \times 10^{-5} \mathrm{C} \mathrm{m}^{-3}$ \\
\hline 1200 & $0,85 \times 10^{-5} \mathrm{C} \mathrm{m}^{-3}$ & $0,95 \times 10^{-5} \mathrm{C} \mathrm{m}^{-3}$ \\
\hline
\end{tabular}

décroissante du temps. Le rapport $\left(q_{\mathrm{v}}\right)_{\text {inst }} /\left(q_{\mathrm{v}}\right)_{\mathrm{st}}$ des densités de charge convectée au début d'écoulement et en régime stationnaire d'électrisation (pour un nombre de Reynolds donné) augmente quand la résistivité augmente. Pour un tube de $1 \mathrm{~m}$ de longueur et $1 \mathrm{~mm}$ de diamètre intérieur et $R_{\mathrm{e}}=400$, ce rapport vaut 1 pour une résistivité de 3 à $4 \times 10^{11} \Omega \mathrm{m}$, il vaut environ 6 pour une résistivité de $7 \times 10^{11} \Omega \mathrm{m}$ et devient 14 pour du cyclohexane sans additif (résistivité $1.7 \times 10^{12} \Omega \mathrm{m}$ ).

La valeur instantanée de la densité volumique de charge convectée est indépendante du nombre de Reynolds, elle varie comme $R^{-2}$ où $R$ est le rayon du tube. Ses variations suivent donc bien la relation théorique $q_{\mathrm{v}}=8 \varepsilon \zeta / R^{2}$.

La valeur instantanée de la densité de charge convectée par différentes solutions est reportée sur la figure 7 en fonction de leur résistivité. Elles corres-

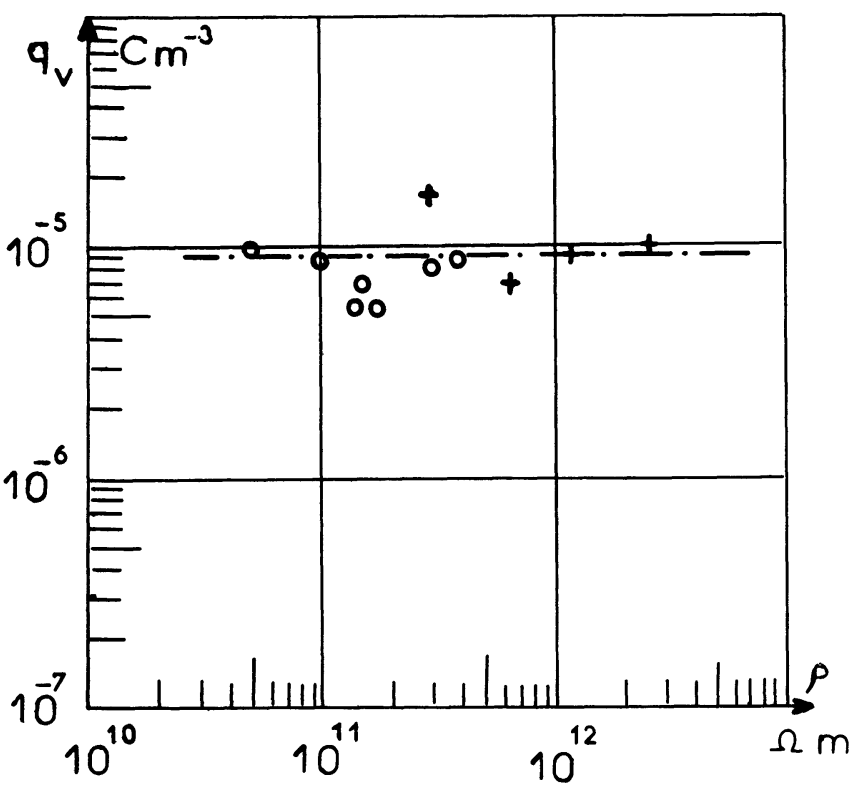

Fig. 7. - Densité de charge convectée $q_{\mathrm{v}}$ en fonction de la résistivité de la solution. $R_{\mathrm{e}}=400 ; R=0,5 \mathrm{~mm}$; longueur du tube $=3 \mathrm{~m}$. (+) Solutions CH + TiAP. (O) Solutions $\mathrm{CH}+\mathrm{TiAP}+$ TIA.

[Resistivity dependence of the convected charge density $q_{\mathrm{v}}$. $R_{\mathrm{e}}=400 ; R=0.5 \mathrm{~mm}$; tube length $=3 \mathrm{~m}$. (+) TiAP in $\mathrm{CH}$; (O) TiAP + TIA in CH.] pondent à nos essais sur des solutions cyclohexane, TiAP et TIA (triisoamylamine), et sur les solutions cyclohexane et TiAP. Les résultats sont assez dispersés, on note pour ces solutions de résistivité assez élevée une médiocre reproductibilité des expériences. Pour ces deux types de solutions, $q_{\mathrm{v}}$ vaut environ $9 \times 10^{-6}$ $\mathrm{C} \mathrm{m}^{-3}$ (rayon du tube $=0,5 \mathrm{~mm}$ ) et peut être considérée comme indépendante de la résistivité de la solution.

L'étude du régime transitoire permet de confirmer que la valeur $\left(q_{\mathrm{v}}\right)_{\text {inst }}$ correspond bien à la distribution d'équilibre de la double couche dans la section de sortie du tube. Tout d'abord, le régime transitoire a une cause électrique et non une cause mécanique : il n'est observé qu'avec des solutions de résistivité élevée et la durée du régime transitoire est très grande devant le temps de mise en vitesse du liquide calculée d'après Bachelor [26].

La durée $T_{0}$ du régime transitoire d'électrisation est donnée sur la figure 8 pour des solutions de différentes résistivités $\rho$ s'écoulant dans des tubes de $3 \mathrm{~m}$ de longueur et $1 \mathrm{~mm}$ de diamètre intérieur. Pour des résistivités inférieures à 3 à $4 \times 10^{11} \Omega \mathrm{m}$, il n'y a plus de régime transitoire d'électrisation; pour des valeurs supérieures à $4 \times 10^{11} \Omega \mathrm{m}, T_{0}$ varie comme $\rho^{-1 / 2}$.

Pour une résistivité donnée, $T_{0}$ varie comme $R_{\mathrm{e}}^{-1}$ et les mesures de $T_{0}$ avec deux tubes de diamètre intérieur 0,66 et $1 \mathrm{~mm}$ montrent une variation en $R^{2}$.

L'ensemble de ces observations argumente l'interprétation suivante du régime transitoire : avant la mise en écoulement du liquide, celui-ci a séjourné environ 10 min dans le tube, et la distribution de charges

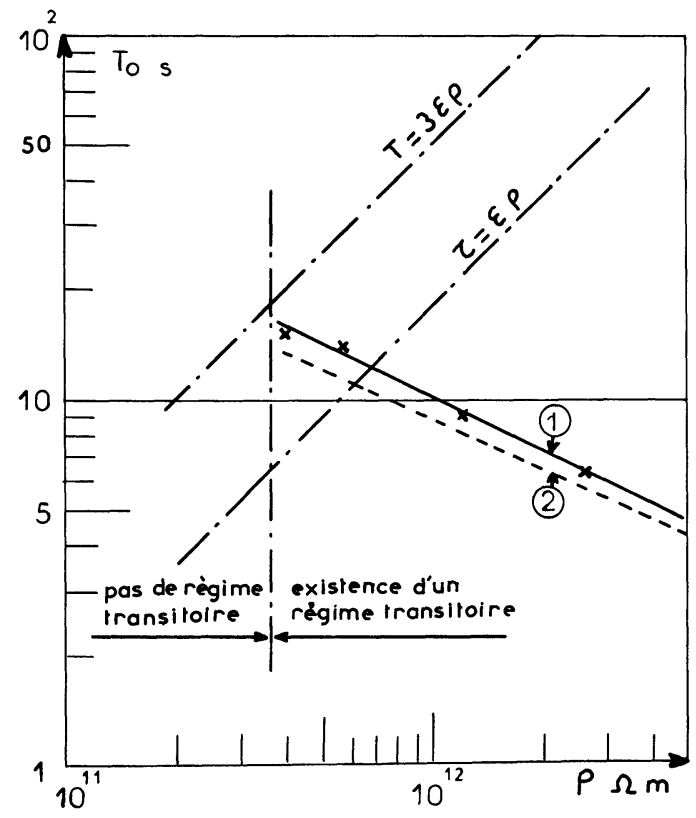

Fig. 8. - Variation de la durée $T_{0}$ du régime transitoire d'électrisation en fonction de la résistivité des solutions $\mathrm{CH}+$ TiAP. $L=3 \mathrm{~m}, R=0,5 \mathrm{~mm}$. 1 . Courbe expérimentale. 2. Courbe théorique.

[Resistivity dependence of the transient duration $T_{0}$. $L=3 \mathrm{~m} ; R=0.5 \mathrm{~mm}$. 1. Experimental curve. 2. Theoretical curve.] 
à l'interface est à l'équilibre. A l'ouverture de la vanne, le liquide sous pression entraîne la couche diffuse qui a sa distribution d'équilibre, la densité de charge convectée vaut $\left(q_{\mathrm{v}}\right)_{\text {inst }}$. Soit $T_{0}$ le temps mis par une particule liquide sortant du tube à la distance $L_{\mathrm{D}}$ (longueur de Debye) de la paroi pour parcourir toute la longueur du tube. Si ce temps $T_{0}$ est petit devant le temps de relaxation $\varepsilon \rho$ du liquide, la couche diffuse n'a pas le temps de s'établir dans le liquide constamment renouvelé, et la densité $q_{\mathrm{v}}$ de charge convectée pour $t>T_{0}$ décroît dans le temps jusqu'à une valeur qui peut être beaucoup plus faible que $\left(q_{\mathrm{v}}\right)_{\text {inst }}$ si la résistivité et le nombre de Reynolds sont grands. Sur la figure 8, on constate qu'il y a disparition du régime transitoire quand $T_{0}=3 \varepsilon \rho$. D'après notre interprétation, $T_{0}$ serait le temps mis par un élément de liquide situé à la distance $L_{\mathbf{D}}$ de la paroi du tube pour parcourir le tube. En régime laminaire, l'expression de $T_{0} \mathrm{~s}$ 'écrit : $T_{0}=L \rho_{\mathrm{v}} R^{2} / 2, R_{\mathrm{e}} \eta\left(K \varepsilon \phi_{0} \rho\right)^{1 / 2}$, où $\rho_{\mathrm{v}}$ est la masse volumique du liquide, $K$ la mobilité ionique et $\phi_{0}=$ $k T / e_{0}$. Cette expression prévoit bien les variations de $T_{0}$ avec $R^{2}, R_{\mathrm{e}}^{-1}$ et $\rho^{-1 / 2}$ observées expérimentalement.

Les valeurs $\left(q_{\mathrm{v}}\right)_{\text {inst }}$ correspondent donc à la distribution stationnaire de la double couche dans la section de sortie du tube et sont les valeurs qui seraient obtenues avec un tube infiniment long.

4.5 Remarque. - Détermination de $\zeta$ dans le cas de solutions résistantes $\left(L_{\mathrm{D}} \gtrsim R\right)$.

Lorsque la résistivité des solutions $\mathrm{CH}+$ TiAP passe de $3 \times 10^{11} \Omega \mathrm{m}$ à $3 \times 10^{12} \Omega \mathrm{m}$, la longueur de Debye varie de 48 à $132 \mu \mathrm{m}$, et le rapport $L_{\mathrm{D}} / R$ de $9,6 \times 10^{-2}$ à 0,26 pour $R=0,5 \mathrm{~mm}$. Pour $L_{\mathrm{D}} / R \sim 1$, l'expression $q_{\mathrm{v}}=8 \varepsilon \zeta / R^{2}$ n'est plus valable, $q_{\mathrm{v}}$ doit être exprimée sous la forme (7).

En supposant que pour les solutions $\mathrm{CH}+$ TiAP, le potentiel $\zeta$ et donc le rapport $q_{\mathrm{B}} / q_{0}$ reste constant et égal à 1,5 , valeur aux faibles résistivités, nous avons tracé sur la figure 9 (courbe 3 ) les variations théoriques de la densité de charge convectée en fonction de la résistivité correspondant à cette valeur de $q_{\mathrm{B}} / q_{0}$, en tenant compte de l'influence du rapport $R / L_{\mathrm{D}}$. Nos résultats expérimentaux sont malheureusement trop dispersés aux résistivités élevées pour nous permettre d'affirmer que le rapport $q_{\mathrm{B}} / q_{0}$ reste constant aux résistivités élevées.

\section{Discussion.}

La densité volumique de charge convectée par écoulement dans des tubes $(L \sim \mathrm{m}, R \sim \mathrm{mm})$ du cyclohexane contenant du TiAP et éventuellement du TIA décroît rapidement quand la résistivité de ces solutions dépasse $5 \times 10^{11} \Omega \mathrm{m}$, la double couche n'ayant pas le temps de s'établir à l'interface métal-liquide. Pour obtenir $q_{\mathrm{v}}$ correspondant à la distribution à l'équilibre de la double couche, il faudrait des longueurs de tube très importantes. Nous avons montré que cette longueur $L$ pouvait être évaluée à l'aide de la relation
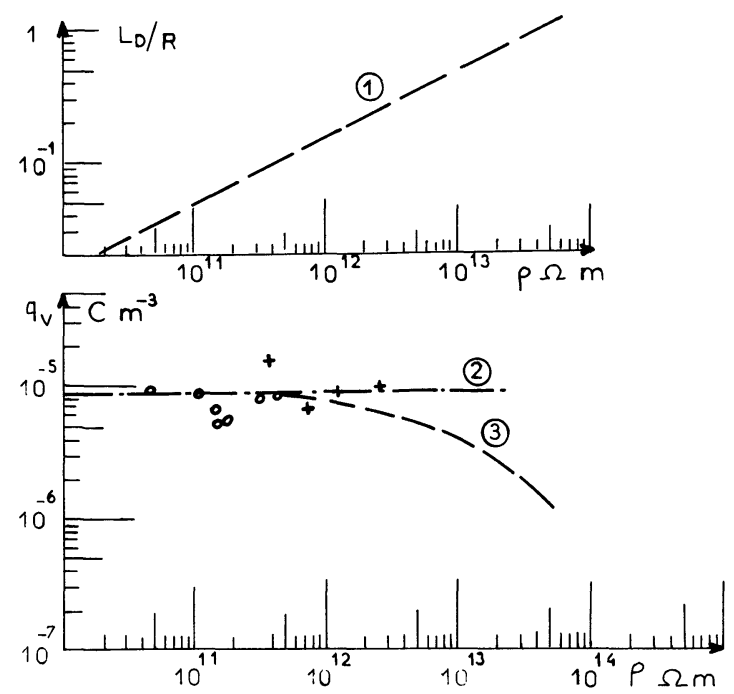

Fig. 9. - Variation du rapport $L_{\mathrm{D}} / R$ avec la résistivité du cyclohexane (courbe 1). Variation de la densité volumique de charges convectées en fonction de la résistivité du liquide (courbe 2). Courbe théorique $q_{\mathrm{v}}(\rho)$ calculée avec $q_{\mathrm{B}} / q_{0}=1,5$.

[Resistivity dependence of the ratio $L_{\mathrm{D}} / R$ in $\mathbf{C H}$ (curve 1). Resistivity dependence of the convected charge density (curve 2). Theoretical curve (3) calculated with $q_{\mathrm{B}} / q_{0}=1.5$.]

$3 \varepsilon \rho=L / U\left(L_{\mathrm{D}}\right)$ où $U\left(L_{\mathrm{D}}\right)$ est la vitesse du liquide à la distance $L_{\mathrm{D}}$ de la paroi du tube. A titre d'exemple, pour un liquide de résistivité $3 \times 10^{12} \Omega \mathrm{m}, \varepsilon_{\mathrm{r}}=2$, s'écoulant dans un tube de rayon $R=0,5 \mathrm{~mm}$, $R_{\mathrm{e}}=100$, on obtient $L=22 \mathrm{~m}$.

La densité volumique de charge convectée peut être plus facilement obtenue par des essais sur des tubes de quelques mètres de longueur à condition d'étudier le régime transitoire d'électrisation; pendant les premiers instants de l'écoulement du liquide, celui-ci entraîne les charges correspondant à une double couche établie.

En procédant ainsi dans les solutions de TiAP dans le cyclohexane, nous mesurons des densités volumiques $q_{\mathrm{v}}$ de charges convectées de l'ordre de 9 à $10 \times 10^{-6} \mathrm{C} \mathrm{m}^{-3}$ pour un rayon du tube $R=0,5 \mathrm{~mm}$, ce qui correspond à un potentiel $\zeta$ de $18 \mathrm{mV}$ environ. Cette valeur semble dépendre très peu de la résistivité de la solution qui dans nos expériences passe de $10^{+11}$ à $3 \times 10^{+12} \Omega \mathrm{m}$.

La densité volumique $q_{\mathrm{B}}$ de charge à la distance $x_{\mathrm{B}}=72 \AA$ de la paroi calculée à partir de $\zeta$ vaut $q_{\mathrm{B}}=1,5 q_{0}$; dans la gamme de résistivité étudiée, il y a proportionnalité entre la densité volumique de charges près de la paroi $\left(q_{\mathrm{B}}\right)$ et celle des ions positifs ou négatifs loin de la paroi $\left(q_{0}\right)$.

Enfin, on peut calculer la densité volumique $q_{\mathrm{p}}$ de charges à la distance $x_{\mathrm{A}}=5 \AA$ du métal, prise comme distance minimum d'approche des ions du TiAP; à partir de la valeur de $q_{\mathrm{B}}$, on obtient $q_{\mathrm{p}}=10^{6} q_{0}$. En raison de la force-image, la densité volumique de charge croît très rapidement quand on s'approche du métal. 


\section{Conclusion.}

Notre étude de l'électrisation du cyclohexane contenant du TiAP confirme l'idée généralement admise que l'électrisation par écoulement d'un liquide résulte de l'entraînement de la couche diffuse qui s'établit à l'interface métal-liquide.

En régime laminaire dans un tube métallique, en prenant certaines précautions expérimentales (étude du régime transitoire), la densité moyenne de charge convectée varie comme $R^{-2}(R=$ rayon du tube) et est indépendante du nombre de Reynolds. D'autre part, l'électrisation partielle de liquides résistants s'écoulant dans des tubes de faible longueur est également bien décrite par un modèle basé sur la couche diffuse. Nous avons également montré que le temps d'établissement de cette couche est de l'ordre du temps $\varepsilon \rho$ de relaxation du liquide.

\section{Remerciements.}

Les auteurs remercient Monsieur Gérard Touchard, pour ses nombreux conseils lors du choix du montage expérimental et pour les calculs numériques qu'il a bien voulu effectuer pour eux.

\section{Bibliographie}

[1] Richter, M. M., R. Oppeheim (Verlag, Berlin) 1893.

[2] KLINKENBERG, A., VAN DER MinNe, J. L., Electrostatics in the petroleum industry (Elsevier, Amsterdam) 1958.

[3] SchöN, G., Handbuch der Raumexplosion, H. H. Freytag (Verlag Chem., Weinheim) 1965.

[4] Gibson, N., Proc. I.P. Conference no 11, Static Electrification, 1971, p. 71.

[5] Strawson, H., Lyle, A. R., 2rd Int. Conf. on Electrical Safety in Hazardous Environments, I.E.E., 1975.

[6] LeONARD, J. T., J. Electrostat. 10 (1981) 17.

[7] BRIGHT, A. W., J. Electrostat. 4 (1977)/(1978) 131.

[8] Denat, A., Gosse, B., Gosse, J. P., J. Electrostat. 7 (1979) 205.

[9] Felici, N., Gosse, J. P., Solofomboahangy, A., 7th Int. Conf. on Conduction and Breakdown in Dielectric Liquids, Berlin, 1981.

[10] Davies, J. T., RideAl, E. K., Interfacial phenomena (Acad. Press, New York) 1963.

[11] Delahay, P., Double layer and electrode kinetics (Interscience Publishers) 1965, p. 33.

[12] Robinson, R. A., Stokes, R. H., Electrolyte solutions (Butterworths, London) 1959.

[13] Felici, N., Gosse, J. P., Solofomboahangy, A., J. Electrostat. 12 (1982) 369.
[14] TOUChARD, G., J. Electrostat. 5 (1978) 463.

[15] Touchard, G., Dumargue, P., J. Electroanal. Chem. 88 (1978) 387.

[16] Rutgers, A. J., De Smet, M., De Myer, G., Trans. Faraday Soc. 53 (1957) 393.

[17] GibBins, J. C., Electroanal. Chim. Interfacial Electrochem. 25 (1970) 497.

[18] Touchard, G., 6th Int. Conf. on Conduction and Breakdown in Dielectric Liquids, Rouen (Ed. Frontières, Dreux) 1978, p. 95.

[19] Touchard, G., Thèse de Doctorat d'Etat, Poitiers, 1978.

[20] Goodfellow, H. D., Graydon, N. F., Chem. Eng. Sci. 23 (1968) 1267.

[21] Luus, R., May, Z., Graydon, N. F., Can. J. Chem. Eng. 45 (1967) 17.

[22] Leonard, J. L., Bogardus, H. F., N.L.R. Report 8021, Washington, 1976.

[23] Kraus, C. A., Fuoss, R. M., J. Am. Chem. Soc. 55 (1933) 23.

[24] Fuoss, R. M., Kraus, C. A., J. Am. Chem. Soc. 55 (1933) 2387.

[25] Koszman, I., Gavis, J., Chem. Eng. Sci. 17 (1962) 1023.

[26] BACHELOR, G., An introduction to fluid dynamics (Cambridge University Press) 1970. 